\title{
Data-aided Channel Estimation for Turbo-PIC MIMO Detectors
}

\author{
Mohammad-Ali Khalighi, Joseph J. Boutros, and Jean-François Hélard
}

\begin{abstract}
We consider data-aided channel estimation for multiple input multiple output (MIMO) systems when iterative parallel interference cancellation (PIC) is performed for signal detection. We compare some data-aided channel estimation methods based on expectation maximization (EM) algorithm or on hard estimated transmit symbols. In particular, we propose a modified EM-based approach and show that when few iterations are to be performed, it provides considerable performance improvement.
\end{abstract}

Index Terms-MIMO systems, channel estimation, iterative detection, Expectation Maximization (EM), interference cancellation, turbo-PIC.

\section{INTRODUCTION}

$\mathbf{M}$ ULTIPLE input multiple output (MIMO) systems can potentially provide very high data rates in a richscattering propagation medium [1]. In the case of coherent signal detection at receiver, channel estimation is necessary. The classical approach of time-multiplexed pilots and data results in an important loss in spectral efficiency for large number of transmit antennas and when channel has rapid variations [2]. Hence, it is of interest to transmit few pilot symbols and to use data-aided (also called semi-blind) channel estimation schemes that make use of data symbols in addition to pilots. In this work, we consider iterative signal detection based on parallel interference cancellation (PIC) [3] and study data-aided channel estimation based on the expectation maximization (EM) algorithm [4]. We first consider the classical implementation of EM, presented in [5], and propose an appropriate formulation of EM under turbo-PIC detection. We next propose a modification to the classical EM algorithm that leads to a better convergence at the receiver. We also study a simple data-aided approach, called Th-HD (for Thresholded Hard Decisions) and initially proposed in [3], that uses reliable detected data symbols in channel estimation in the same way as pilots. Frequency non-selective, uncorrelated quasi-static Rayleigh fading and QPSK modulation are considered.

\section{Signal TRAnsmission And Detection}

At the transmitter, bit-interleaved coded modulation is used using a non-recursive non-systematic convolutional (NRNSC) code. After being randomly interleaved, encoded data bits are mapped to symbols $x$ that are power normalized, and then

Manuscript received October 17, 2005. The associate editor coordinating the review of this letter and approving it for publication was Prof. Jing Li.

M.-A. Khalighi is with the Institut Fresnel, Marseille, France (e-mail: Ali.Khalighi@ieee.org).

J. J. Boutros is with GET/Télécom Paris (ENST), France. (e-mail: Boutros@enst.fr).

J.-F. Hélard is with the Institut d'Électronique et de Télécommunications de Rennes, France (e-mail: Jean-Francois.Helard@insa-rennes.fr).

Digital Object Identifier 10.1109/LCOMM.2006.05019.

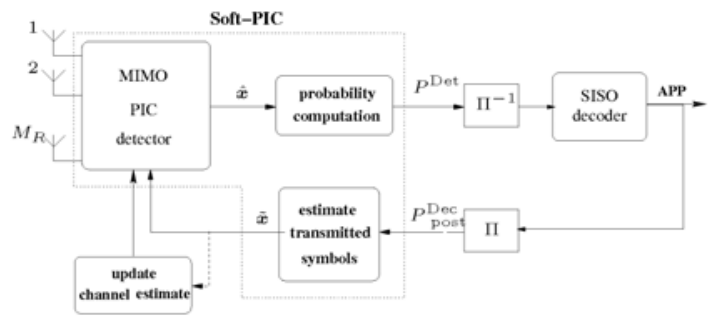

Fig. 1. Block diagram of turbo-PIC detector.

transmitted on $M_{T}$ antennas. We call a vector $\boldsymbol{x}$ of $M_{T}$ symbols a compound symbol. Let $\boldsymbol{H}$ be the channel matrix of dimension $\left(M_{R} \times M_{T}\right)$ and $\mathrm{n}$ the vector of complex circularly symmetric AWGN of variance $\sigma_{n}^{2}$. The vector $\boldsymbol{y}$ of received signals on $M_{R}$ antennas at a time sample $n$ is:

$$
\boldsymbol{y}[n]=\boldsymbol{H} \boldsymbol{x}[n]+\mathrm{n}[n] .
$$

At the receiver, iterative symbol detection and channel decoding is done as shown in Fig.1. The detector is composed of two main blocks of Soft-PIC and maximum a posteriori (MAP) soft-input soft-output (SISO) decoder. Soft-PIC is described in detail in [3], [6]. At the first iteration, PIC detection is equivalent to minimum mean-square error (MMSE) filtering. The detected symbol of the transmit antenna \#k is:

$$
\hat{x}_{k}^{(1)}=\boldsymbol{h}_{k}^{\dagger}\left(\boldsymbol{H} \boldsymbol{H}^{\dagger}+\sigma_{n}^{2} \boldsymbol{I}\right)^{-1} \boldsymbol{y} .
$$

$\boldsymbol{h}_{k}$ is the $k^{t h}$ column of $\boldsymbol{H}, .^{\dagger}$ denotes conjugate-transpose, and $\boldsymbol{I}$ is the Identity matrix. In next iterations, we estimate the transmitted symbols using the a posteriori probabilities (APP) at the SISO decoder output. These estimates $\tilde{\boldsymbol{x}}$ are used in PIC detector to reduce the co-antenna interference (CAI) before zero-forcing filtering. We use a suboptimal filtering proposed in [3] that assumes almost perfect CAI cancellation. Let $\boldsymbol{H}_{k}$ of dimension $\left(M_{R} \times\left(M_{T}-1\right)\right)$ be $\boldsymbol{H}$ with its $k^{\text {th }}$ column removed, and $\tilde{\boldsymbol{x}}_{k}^{(m-1)}$ of dimension $\left(\left(M_{T}-1\right) \times 1\right)$ the vector of estimated symbols at iteration $(m-1)$ with its $k^{\text {th }}$ entry removed. The detected $k^{t h}$ symbol at iteration $m$ is:

$$
\hat{x}_{k}^{(m)}=\left(\boldsymbol{h}_{k}^{\dagger} \boldsymbol{h}_{k}\right)^{-1} \boldsymbol{h}_{k}^{\dagger}\left(\boldsymbol{y}-\boldsymbol{H}_{k} \tilde{\boldsymbol{x}}_{k}^{(m-1)}\right) \text {. }
$$

\section{Channel estimation}

Let $N_{p s}$ and $N_{d s}$ be respectively the number of pilot and data compound symbols in a frame, corresponding to $N_{p}$ and $N_{d}$ pilot and data bits. For instance, with $B$ bits per symbol, $N_{p}=N_{p s} B M_{T}$. Also, let $N_{s}=N_{d s}+N_{p s}$. The maximum likelihood channel estimate based only on pilot symbols is given in (4) where $\boldsymbol{x}_{p}$ denotes a compound pilot symbol, i.e., a vector of $M_{T}$ pilot symbols.

$$
\hat{\boldsymbol{H}}^{p}=\left(\sum_{n=1}^{N_{p s}} \boldsymbol{y}[n] \boldsymbol{x}_{p}^{\dagger}[n]\right)\left(\sum_{n=1}^{N_{p s}} \boldsymbol{x}_{p}[n] \boldsymbol{x}_{p}^{\dagger}[n]\right)^{-1}
$$




\section{A. Classical EM-based data-aided estimation}

The classical formulation of EM that we call Mix-EM (or Mixing-EM) considers the ensemble of pilot and data symbols as missing data [5]. In this way, after simplifying the expectation and maximization steps, we come to the following estimation update equation for $\boldsymbol{H}$ at iteration $m>1$ [5]:

$$
\hat{\boldsymbol{H}}=\overline{\boldsymbol{R}}_{y x} \overline{\boldsymbol{R}}_{x}^{-1}
$$

where

$$
\begin{aligned}
\overline{\boldsymbol{R}}_{y x} & =\sum_{n=1}^{N_{s}} \sum_{q=1}^{Q} \boldsymbol{y}[n] \boldsymbol{x}_{q}^{\dagger} A P P_{n}\left(\boldsymbol{x}_{q}\right) \\
\overline{\boldsymbol{R}}_{x} & =\sum_{n=1}^{N_{s}} \sum_{q=1}^{Q} \boldsymbol{x}_{q} \boldsymbol{x}_{q}^{\dagger} A P P_{n}\left(\boldsymbol{x}_{q}\right)
\end{aligned}
$$

$Q=2^{B M_{T}}$ and $\boldsymbol{x}_{q}$ is the $q^{\text {th }}$ among $Q$ possible compound symbols whose probability of transmission $A P P_{n}\left(\boldsymbol{x}_{q}\right)$ is calculated using the previous APPs at the SISO decoder output:

$$
A P P_{n}\left(\boldsymbol{x}_{q}\right) \propto \prod_{i=1}^{B M_{T}} P_{\text {post }}^{\mathrm{Dec}}\left(c_{q, i}\right)
$$

$P_{\text {post }}^{\mathrm{Dec}}\left(c_{q, i}\right)$ is the APP corresponding to the $i^{t h}$ bit of $\boldsymbol{x}_{q}, c_{q, i} .{ }^{1}$ For the first iteration, we use $\hat{\boldsymbol{H}}=\hat{\boldsymbol{H}}^{p}$ from (4). The computational complexity of (6) and (7) increases exponentially with $B M_{T}$. However, it can be shown that $\sum_{q=1}^{Q} \boldsymbol{x}_{q} A P P\left(\boldsymbol{x}_{q}\right)=$ $\tilde{\boldsymbol{x}}$. But $\tilde{\boldsymbol{x}}$ is already computed in Soft-PIC and there is no need to compute it again. Now, (6) and (7) can be written as:

$$
\begin{gathered}
\overline{\boldsymbol{R}}_{y x}=\sum_{n=1}^{N_{s}} \boldsymbol{y}[n] \tilde{\boldsymbol{x}}^{\dagger}[n] \\
\overline{\boldsymbol{R}}_{x}(i, j)=\left\{\begin{array}{cc}
N_{s} & ; i=j \\
\sum_{n=1}^{N_{s}} \tilde{x}_{i}[n] \tilde{x}_{j}^{*}[n] & ; i \neq j
\end{array}\right.
\end{gathered}
$$

$\tilde{x}_{i}[n]$ is the $i^{t h}$ entry of the vector $\tilde{\boldsymbol{x}}[n]$. So, EM is implemented in the body of Soft-PIC and in a much simpler way.

\section{B. Modifying EM formulation}

Using (1) and (9), we can write $\overline{\boldsymbol{R}}_{y x}=\boldsymbol{H} \boldsymbol{R}_{x}^{\prime}+\boldsymbol{\eta}$, where

$$
\boldsymbol{R}_{x}^{\prime} \triangleq \sum_{n=1}^{N_{s}} \boldsymbol{x}[n] \tilde{\boldsymbol{x}}^{\dagger}[n] \quad, \quad \boldsymbol{\eta}=\sum_{n=1}^{N_{s}} \mathrm{n}[n] \tilde{\boldsymbol{x}}^{\dagger}[n] .
$$

$\boldsymbol{\eta}$, the matrix of weighted noise samples, has the autocovariance matrix $\sigma_{n}^{2} \boldsymbol{R}_{x}^{\prime \prime}$, where $\boldsymbol{R}_{x}^{\prime \prime}=\sum_{n=1}^{N_{s}} \tilde{\boldsymbol{x}}[n] \tilde{\boldsymbol{x}}^{\dagger}[n]$. Now from (5) $\hat{\boldsymbol{H}}$ can be written as:

$$
\hat{\boldsymbol{H}}=\boldsymbol{H} \boldsymbol{R}_{x}^{\prime} \overline{\boldsymbol{R}}_{x}^{-1}+\boldsymbol{\eta} \overline{\boldsymbol{R}}_{x}^{-1} .
$$

We see from (10) and (11) that $\boldsymbol{R}_{x}^{\prime} \neq \overline{\boldsymbol{R}}_{x}$, and hence, $\hat{\boldsymbol{H}}$ is a biased estimate. This is due to the fact that $\tilde{\boldsymbol{x}} \neq \boldsymbol{x}$ except at high enough SNR and at concluding iterations where the bias becomes negligible. The bias can degrade the receiver performance by affecting the detector convergence.

\footnotetext{
${ }^{1}$ Equation (8) gives an approximation of the exact $\quad{ }_{n}\left(\boldsymbol{x}_{q}\right)$ and is suboptimal in terms of bit-error-rate and convergence speed, but it is much less complex than the exact calculation of APPs. For more details, see [5].
}

To remove the bias, we consider separately the unbiased pilotonly-based estimate $\hat{\boldsymbol{H}}^{p}$ and combine it with $\hat{\boldsymbol{H}}^{d}$, the databased estimate via EM, to obtain $\hat{\boldsymbol{H}}$. In the sequel, we consider matrices $\overline{\boldsymbol{R}}_{x}, \boldsymbol{R}_{x}^{\prime}$, and $\boldsymbol{R}_{x}^{\prime \prime}$ calculated only over data symbols, i.e., with the summations taken on $N_{d s}$ instead of $N_{s}$. One solution is to remove the bias of $\hat{\boldsymbol{H}}^{d}$ by Wiener or inverse filtering, and then to combine the resulting estimate with $\hat{\boldsymbol{H}}^{p}$. This approach, called RB-EM in [6] (Removed Biased-EM), is computationally complex and does not provide satisfying results either [6]. A more appropriate solution that we present here, assumes large enough $N_{d s}$ and negligible CAI on $\tilde{\boldsymbol{x}}$. Thus, neglecting the off-diagonal terms of $\overline{\boldsymbol{R}}_{x}, \boldsymbol{R}_{x}^{\prime}$, and $\boldsymbol{R}_{x}^{\prime \prime}$, from (12) we obtain:

$$
\hat{\boldsymbol{H}}^{d} \approx \frac{1}{N_{d s}} \boldsymbol{H} \operatorname{diag}\left(\sum_{n=1}^{N_{d s}} x_{i}[n] \tilde{x}_{i}^{*}[n]\right)+\boldsymbol{\eta}^{\prime \prime}
$$

where $\operatorname{diag}($.$) denotes a diagonal matrix with its (i, i)$ th entry given, and $\boldsymbol{\eta}^{\prime \prime}=\frac{1}{N_{d s}} \boldsymbol{\eta}$. The $(i, j)$ th entry of $\hat{\boldsymbol{H}}^{d}$ is then,

$$
\hat{H}_{i j}^{d}=\alpha_{j} H_{i j}+\eta_{i j}^{\prime \prime} \text { where } \alpha_{j} \triangleq \frac{1}{N_{d s}} \sum_{n=1}^{N_{d s}} x_{j}[n] \tilde{x}_{j}^{*}[n] .
$$

We deduce from (14) that we should combine $\hat{\boldsymbol{H}}^{d}$ and $\hat{\boldsymbol{H}}^{p}$ columnwise by optimal coefficients $a_{j}$ and $b_{j}$ :

$$
\hat{\boldsymbol{h}}_{j}=a_{j} \hat{\boldsymbol{h}}_{j}^{d}+b_{j} \hat{\boldsymbol{h}}_{j}^{p}
$$

The optimization criterion is to minimize the mean square estimation error variance, i.e., $\min \left\{\left|a_{j}\right|^{2} \hat{\sigma}_{j, d}^{2}+\left|b_{j}\right|^{2} \hat{\sigma}_{j, p}^{2}\right\}$, subject to unbiased estimation, i.e., $a_{j} \alpha_{j}+b_{j}=1$. Here $|$. stands for complex modulus, $\hat{\sigma}_{j, d}^{2}=\frac{1}{N_{d s}^{2}} R_{x j j}^{\prime \prime}$ is the variance of $\boldsymbol{\eta}_{j}^{\prime \prime}$ entries, and $\hat{\sigma}_{j, p}^{2}=\sigma_{n}^{2} / N_{p s}$ for mutually orthogonal pilot sequences. Using Lagrange multipliers method we obtain:

$$
a_{j}=\frac{\alpha_{j}^{*}}{\left|\alpha_{j}\right|^{2}+\frac{N_{p s}}{N_{d s}^{2}} R_{x j j}^{\prime \prime}}, b_{j}=\frac{\frac{N_{p s}}{N_{d s}^{2}} R_{x j j}^{\prime \prime}}{\left|\alpha_{j}\right|^{2}+\frac{N_{p s}}{N_{d s}^{2}} R_{x j j}^{\prime \prime}} .
$$

We call this solution MU-EM (Modified Unbiased-EM) [6]. To calculate $\alpha_{j}$, we replace $x_{j}$ in (14) by the hard decision on $\tilde{x}_{j}$, assuming a low enough error probability. Note that at low SNR and in first iterations, we have $\alpha_{j} \approx 0$ and $R_{x j j}^{\prime \prime} \approx 0$, and so, $\hat{\boldsymbol{H}} \approx \hat{\boldsymbol{H}}^{p}$. On the other hand, at high SNR and in concluding iterations, we have $\alpha_{j} \approx 1$ and $R_{x j j}^{\prime \prime} \approx N_{d s}$.

\section{Th-HD estimation method}

With Th-HD, the APPs at the SISO decoder output are compared to a threshold $P_{T H}$ in order to choose reliable detected data symbols. If $\mathrm{APP}>P_{T H}$ the hard decision 1 is assigned to the corresponding bit, and if $\mathrm{APP}<\left(1-P_{T H}\right)$, the hard decision 0 is assigned to it. Hard-detected compound symbols are then used in channel estimation like pilots. Simulation results (not presented) show poor dependence of the Th-HD performance to the choice of $P_{T H}$ and an optimal $P_{T H}$ close to 0.5 . This comes to using hard estimates of data symbols in channel estimation in the same way as pilots. 


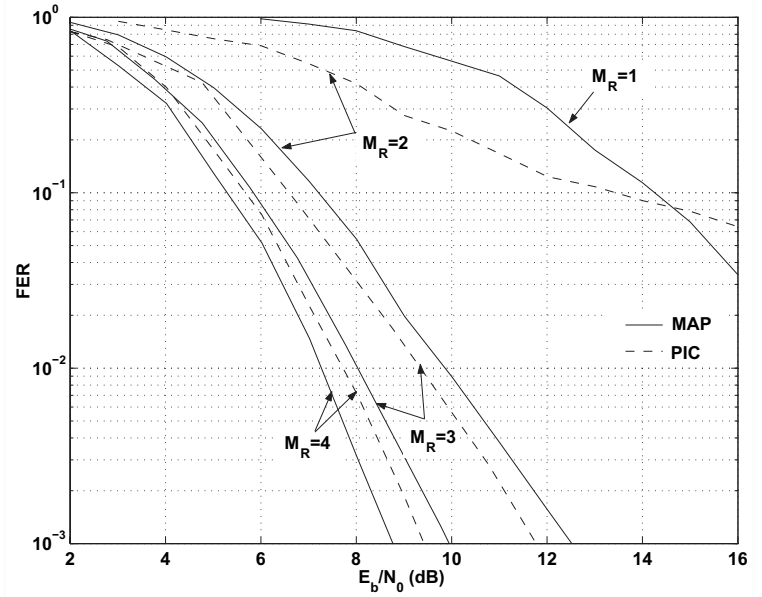

Fig. 2. Comparing turbo-PIC and turbo-MAP; $T=4$, NRNSC code $(57)_{8}, \quad d=512$, perfect CSI, fifth iteration of the detector.

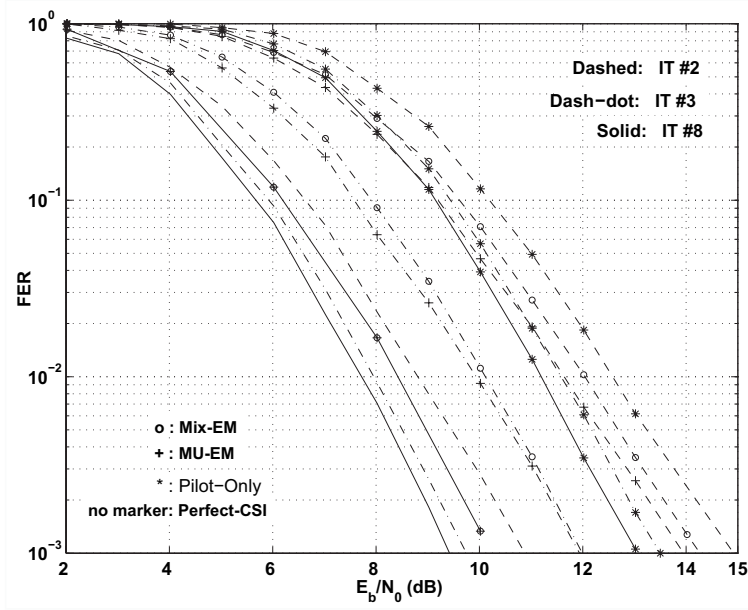

Fig. 3. Comparing perfect CSI and pilot-only, Mix-EM, and MU-EM estimation methods for second, third, and eighth iteration; $T=R=4$, NRNSC code $(57)_{8}, \quad d=512, \quad p=40$.

\section{Simulation Results}

Almost the same $N_{d s}$ is considered for different $M_{T}$. Optimal pilot sequences are used with $N_{p s}$ a little larger than the limit of identifiability $B M_{T}$. Let us first compare the performances of the turbo-PIC and turbo-MAP [5] detectors when perfect channel state information (CSI) is available at receiver. Fig. 2 contrasts frame error rate (FER) curves for $M_{T}=4$ and $M_{R} \leq M_{T}$. Receiver array gain is included in $E_{b} / N_{0}$. We see that thanks to iterative detection, our PICbased detector works efficiently for $M_{R}=3$ with a reasonable loss compared to turbo-MAP. For $M_{R}<3$, the detector does not provide satisfying performance although it does not diverge. Without coming to an analytical proof, we verified that turbo-PIC works efficiently for $M_{R}>M_{T} / 2$ where it would be preferred to turbo-MAP due to its considerably lower complexity. We also verified that turbo-PIC is slightly less sensitive to channel estimation errors than turbo-MAP.

Fig.3 compares FERs after two, three, and eight iterations for the cases of perfect CSI and estimation based on pilots only, Mix-EM, and MU-EM. For the two former cases, full convergence is attained after five iterations. We see that after

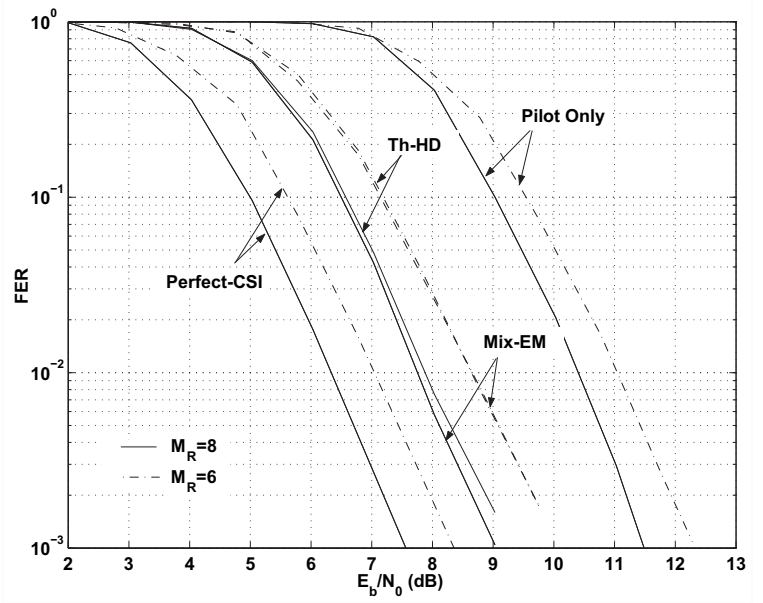

Fig. 4. Comparing Pilot-Only, Mix-EM, and Th-HD channel estimation

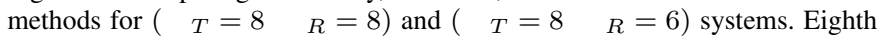
iteration of detector, NRNSC code $(57)_{8}, \quad d=1024, \quad p=160$.

eight iterations, we gain $2.7 \mathrm{~dB}$ in $E_{b} / N_{0}$ at $\mathrm{FER}=10^{-2}$ with Mix-EM compared to pilot-only estimation. Comparing Mix-EM and MU-EM, we notice that for the second and third iterations, MU-EM gives respectively a gain of $0.4 \mathrm{~dB}$ and $0.18 \mathrm{~dB}$ in SNR at FER $=10^{-2}$. However, if we proceed to the eighth iteration, their performances become equivalent. At the first iterations, MU-EM works better than Mix-EM by imposing a low weight to $\hat{\boldsymbol{H}}^{d}$ and by privileging $\hat{\boldsymbol{H}}^{p}$. Fig.4 compares Th-HD and EM-based methods for $M_{T}=8$ and $M_{R}=6,8$ after eight iterations. It is seen that using hard estimated data symbols in channel estimation (Th-HD with $\left.P_{T H}=0.5\right)$ provides almost the same performance as using soft estimates (Mix-EM). However, since soft estimates are already available in turbo-PIC, Mix-EM is preferred.

\section{CONClusion}

We proposed an appropriate formulation for the classical Mix-EM under turbo-PIC detection. We also proposed MUEM that permits a better convergence of the detector and provides interesting performance improvement for small number of iterations. Use of hard estimates of data symbols in channel estimation was shown to provide the same performance as Mix-EM. As a side contribution, we also showed the interest of using turbo-PIC for $M_{R}>M_{T} / 2$.

\section{REFERENCES}

[1] E. Telatar, "Capacity of multi-antenna Gaussian channels," Eur. Trans. Telecomm., vol. 10, pp. 585-595, Nov./Dec. 1999.

[2] B. Hassibi and B. M. Hochwald, "How much training is needed in multiple-antenna wireless links?" IEEE Trans. Inform. Theory, vol. 49, pp. 951-963, Apr. 2003.

[3] M. Sellathurai and S. Haykin, "Turbo-BLAST for wireless communications: theory and experiments," IEEE Trans. Signal Processing, vol. 50, pp. 2538-2546, Oct. 2002.

[4] T. K. Moon, "The expectation-maximization algorithm," IEEE Signal Processing Mag., vol. 13, pp. 47-60, Nov. 1996.

[5] J. Boutros, F. Boixadera, and C. Lamy, "Bit-interleaved coded modulations for multiple-input multiple-output channels," in Proc IEEE ISSSTA'O0, vol. 1, pp. 123-126.

[6] M. A. Khalighi and J. Boutros, "Channel estimation in turbo-BLAST detectors using EM algorithm," in Proc IEEE ICASSP'05, vol. 3, pp. 1037-1040. 